\title{
DISTRIBUTING PLANNING AND CONTROL FOR TEAMS OF COOPERATING MOBILE ROBOTS
}

June 2004

Prepared by

L. E. Parker

Adjunct Distinguished R\&D Staff 
CRADA ORNL 98-0528

DISTRIBUTING PLANNING AND CONTROL FOR TEAMS OF COOPERATING MOBILE ROBOTS

L. E. Parker

Prepared by

OAK RIDGE NATIONAL LABORATORY

P.O. Box 2008

Oak Ridge, Tennessee 37831-6285

managed by

UT-Battelle, LLC

for the

U.S. DEPARTMENT OF ENERGY

under contract DE-AC05-00OR22725 


\title{
Distributed Planning and Control for Teams of Cooperating Mobile Robots ORNL-Caterpillar CRADA 98-0528
}

\author{
Final Report
}

\begin{abstract}
This CRADA project involved the cooperative research of investigators in ORNL's Center for Engineering Science Advanced Research (CESAR) with researchers at Caterpillar, Inc. The subject of the research was the development of cooperative control strategies for autonomous vehicles performing applications of interest to Caterpillar customers. The project involved three Phases of research, conducted over the time period of November 1998 through December 2001. This project led to the successful development of several technologies and demonstrations in realistic simulation that illustrated the effectiveness of our control approaches for distributed planning and cooperation in multi-robot teams.
\end{abstract}

\section{Statement of Objectives}

The primary objectives of this research project were to:

(1) Develop autonomous control technologies to enable multiple vehicles to work together cooperatively,

(2) Provide the foundational capabilities for a human operator to exercise oversight and guidance during the multi-vehicle task execution, and

(3) Integrate these capabilities to the ALLIANCE-based autonomous control approach for multi-robot teams.

These objectives have been successfully met with the results implemented and demonstrated in a near real-time multi-vehicle simulation of up to four vehicles performing mission-relevant tasks.

\section{Benefits to the Funding DOE Office's Mission}

This collaborative research project was of benefit to DOE's basic science objective by significantly advancing the state of the art in cooperative systems. The research resulted in robust cooperative multi-robot teams that are easy to task and control by human controllers. The general cooperative robotic technologies developed under this CRADA are directly applicable to the DOE mission objective of advancing fundamental science. These developed technologies are of a general nature, which also makes them applicable to a wide range of practical applications 
of interest to DOE, such as hazardous waste cleanup and decontamination and decommissioning, to mention a few.

\section{Technical Discussion of Work Performed by All Parties}

The fundamental research development in this project resulting in several contributions to the state of the art in cooperative multi-robot teaming. These technical contributions are summarized below:

- Significant extensions to the Simtool simulator to enable multi-vehicle operations. At the beginning of this project, Caterpillar provided to ORNL a single-robot simulation system, called SIMTOOL, that allows the testing and development of software for robots operating in 3-dimensional environments. SIMTOOL is especially beneficial for certain types of applications, such as surface coal mining, because it allows the simulation of dynamic interactions with and alteration of the terrain, through the use of soil models. ORNL researchers significantly extended this simulation tool to enable: (1) multi-vehicle control, and (2) near real-time simulations.

- Detailed task analysis of two prototypical applications of interest to Caterpillar - surface coal mining and loader-truck-dozer teaming. To fully understand the nature of prototypical surface mining tasks of interest to this project, ORNL researchers performed a detailed task analysis of two key applications. This task analysis identified the following major components of these tasks: organization of control, information requirements, communication, sensing, task distribution, routine performance versus fault recovery, and measures of performance. A document outlining the results and their impact on cooperative control design was produced.

- Development of cooperative control software using the ALLIANCE architecture. Numerous cooperative robot behaviors were designed, programmed, debugged, and demonstrated by ORNL researchers. These robot team behaviors included get-in-slot, dig-carry-dump-return, remove-berm, remove-highwall-overburden, tandem-doze, digout-vehicle, rip-overburden, get-help, and cease-ops-and-return. These were organized and controlled through a higher-level architecture developed by ORNL researchers, called ALLIANCE. Complete missions using up to four autonomous vehicles were successfully demonstrated in simulation using these behaviors.

- Human controller interfaces to cooperative control solution. ORNL researchers developed the Fleet Supervisor Interface (FleetSI), which is a graphical interface for the human operator. FleetSI provides capabilities to the operator controller to input commands to the multi-robot team, to test and experiment with vehicle responses in various situations, as well as to monitor the performance of the vehicles as they are conducting their mission. 


\section{Subject Inventions}

No subject inventions were developed under this project.

\section{Commercialization Possibilities}

A significant portion of the United States' Gross National Product is accounted for in the building, maintaining, and rebuilding of the nation's infrastructure. Industries such as earthmoving, agriculture, mining, waste disposal, aggregates and quarry, forestry, and building construction industries play a role in almost all economic activities. There are numerous motivations for using cooperative robotics in these infrastructure industries. Cost and efficiency are the most critical drivers, where savings of fractions of a second in cycle time can accumulate to millions of dollars of additional production. Cooperating machine systems that are able to self-organize to most efficiently execute a task can greatly increase the ability of an operator to oversee more than one piece of equipment in its operation - a capability of great significance for many industries in which the shortage of skilled workers can lead to project cancellation. Other commercial possibilities for the developed research include increased flexibility, productivity, safety, and consistency. The research in cooperative autonomous teams developed in this CRADA project enables this type of cooperation among multiple pieces of machinery. This research thus has significant potential for revolutionizing the infrastructure industries across the nation.

\section{Plans for Future Collaboration}

The parties involved are very interested in resuming this collaboration at some future date. The results of this project were excellent, and can lead to many possible research extensions in the future. However, at present, due to funding constraints at both Caterpillar and DOE, no definitive plans are in place for resuming this research collaboration.

\section{Conclusions}

This CRADA project was very successful, leading to the accomplishment of the objectives of this research. As a result of this project, new cooperative multi-vehicle control has been demonstrated in a realistic simulation for a prototypical surface mining task. This cooperative control approach has the potential for significantly improving the performance of vehicles in a variety of infrastructure tasks. Significant progress has been made toward a fieldable mixture of human-controlled and autonomous vehicle teams. The intent is to pursue the continuation of this research at some future date, when funding becomes available at both Caterpillar and DOE. 